\title{
CAPACIDADES DE INOVAÇÃO EM SERVIÇOS: UM ESTUDO NOS SUPERMERCADOS EM SANTA CATARINA
}

\section{RESUMO}

O objetivo desta pesquisa consiste em analisar as relações entre as capacidades inovativas, diferenciação de serviços e o desempenho organizacional. Foram pesquisadas as evidências teóricas relativas as organizações que desenvolvem elementos inovadores e melhorar o desempenho organizacional, quando mediadas pela diferenciação de serviços. A metodologia utilizada foi o estudo bibliográfico acerca das variáveis da capacidade inovativa organizacional, os elementos da diferenciação de serviços e os itens de controle do desenvolvimento organizacional. A partir dos estudos teóricos foram possíveis desenvolver 10 proposições destas relações. Sendo as propostas: i) Capacidades inovativas têm relação positiva com desempenho; ii) Capacidades inovativas têm relação positiva com Diferenciação de Serviços na dimensão Processos; iii) Capacidades inovativa têm relação positiva com Diferenciação de Serviços na dimensão Pessoas; iv) Capacidades inovativa têm relação positiva com Diferenciação de Serviços na dimensão Ambientes; v) Diferenciação de Serviços na dimensão Processos tem relação positiva com o desempenho. vi) Diferenciação de Serviços na dimensão Pessoas tem relação positiva com o desempenho; vii) Diferenciação de Serviços na dimensão Ambientes tem relação positiva com o desempenho; viii) Capacidades inovativa têm relação positiva com o desempenho mediado pela diferenciação de serviços em sua dimensão Pessoas; ix) Capacidades inovativa têm relação positiva com o desempenho mediado pela diferenciação de serviços em sua dimensão Processos; e x) Capacidades inovativa têm relação positiva com o desempenho mediado pela diferenciação de serviços em sua dimensão Ambiente.

Palavras-chave: Capacidade Inovativa; Diferenciação de Serviços; Desempenho.

\section{INNOVATION CAPABILITIES IN SERVICES: A STUDY IN SUPERMARKETS IN SANTA CATARINA}

\section{ABSTRACT}

It is common to find organizational research seeking to understand the predictors elements to organizational performance. The objective of this research is to analyze the relationships between innovative capabilities, service differentiation and organizational performance. It has been researched theoretical evidence to support organizations that develop innovative element, therefore its innovative capacity, which is mediated differentiation of services improve performance organizacional.A methodology used was the bibliographical study of the variables of organizational innovative capacity, the element the differentiation of services and organizational development control items. From the theoretical studies it has been possible to develop 10 propositions of these relationships. And proposals: i) innovative capabilities have positive relationship with performance; ii) innovative capabilities have positive relationship with Service Differentiation in the Process dimension; iii) innovative capabilities have positive relationship with Service Differentiation in the People dimension; iv) innovative capabilities have positive relationship with Service Differentiation in environments dimension; v) Service Differentiation in the Process dimension has positive relationship with performance. vi) Service Differentiation in the People dimension has positive relationship with performance; vii) Service Differentiation in environments dimension has positive relationship with performance; viii) innovative capabilities have positive relationship with performance-mediated differentiation of services in its people dimension; ix) innovative capabilities have positive relationship with performance-mediated differentiation of services in their processes dimension; and $\mathrm{x}$ ) innovative capabilities have positive relationship with performance-mediated differentiation of services in its environmental dimension.

Keywords: Innovative Capacity; Differentiation of Services; Performance. 


\section{CAPACIDADES INNOVACIÓN EN LOS SERVICIOS: UN ESTUDIO EN SUPERMERCADOS EN SANTA CATARINA}

\section{RESUMEN}

El objetivo de esta investigación es analizar las relaciones entre la capacidad de innovación, la diferenciación de servicios y rendimiento de la organización. evidencia teórica fue examinada para las organizaciones que desarrollan elementos innovadores y mejorar el desempeño organizacional, cuando mediada por la diferenciación de servicios. La metodología utilizada fue el estudio bibliográfico de las variables de la capacidad innovadora de la organización, los elementos de diferenciación de servicios y elementos de control del desarrollo de la organización. A partir de los estudios teóricos que ha sido posible desarrollar estas relaciones 10 proposiciones. Y las propuestas: i) la capacidad de innovación se relacionan positivamente con el rendimiento; ii) la capacidad de innovación tienen relación positiva con servicio Diferenciación en la dimensión de proceso; iii) capacidad de innovación tienen relación positiva con servicio Diferenciación en la dimensión personas; iv) la capacidad de innovación tienen relación positiva con servicio Diferenciación en entornos dimensión; v) la diferenciación de servicios en la dimensión de proceso tiene una relación positiva con el rendimiento. vi) la diferenciación de servicios en la dimensión gente tiene una relación positiva con el rendimiento; vii) la diferenciación de servicios en dimensión entornos tiene relación positiva con el rendimiento; viii) la capacidad de innovación tienen relación positiva con el rendimiento diferenciación mediada de servicios en su dimensión personas; ix) capacidad de innovación tienen relación positiva con el rendimiento diferenciación mediada de servicios en su dimensión procesos; y x) capacidades innovadoras tienen relación positiva con la diferenciación rendimiento mediada de servicios en su dimensión ambiental.

Palabras clave: La Capacidad Innovadora; La Diferenciación de los Servicios; Rendimiento.

\footnotetext{
${ }^{1}$ Doutorando em Administração pela Universidade do Vale do Itajaí - UNIVALI. Professor de Administração da Universidade Federal de Santa Catarina - UFSC. Brasil. E-mail: mdeluca@linhalivre.net

${ }^{2}$ Doutor em Engenharia de Produção pela Universidade Federal de Santa Catarina - UFSC. Professor da Universidade do Vale do Itajaí - UNIVALI. Brasil. E-mail: claudio.goncalo@univali.br

${ }^{3}$ Doutor em Administração e Turismo pela Universidade do Vale do Itajaí - UNIVALI. Professor do Instituto Federal de Santa Catarina - IFSC. Brasil. E-mail: deosir@ifsc.edu.br

${ }^{4}$ Doutor em Engenharia de Produção pela Universidade Federal de Santa Catarina - UFSC. Professor da Universidade Federal de Santa Catarina - UFSC. Brasil. E-mail: mfpcris@ gmail.com
} 


\section{INTRODUÇÃO}

Rebouças (2008) visualiza o ensaio não como uma forma ou um gênero literário, sendo uma intersecção entre o que é imaginação e a realidade. Desta forma o ensaio não busca exaurir nenhum tema ou assunto, mas sim sua reflexão. Meneghetti (2011), por sua vez, entende o ensaio teórico como a interação da subjetividade com a objetividade entre o sujeito e o objeto. Logo o ensaio teórico não se obtém respostas e afirmações verdadeiras, mas pelas perguntas que orientam os sujeitos.

Para Boava, Macedo e Sette (2012) um ensaio teórico deve conter a dimensão pouco explorada no campo da pesquisa organizacional. Partindo de uma orientação funcionalista de pesquisa, e possuindo em seu ponto central a produção de um saber embasado em relações de causa e efeito.

Com estas permissas, e a percepção de que as organizações atuais estão inseridas em um ambiente munificiente, complexo e bastante dinâmico. Anteriormente, um ou mais recursos poderiam dar uma vantagem competitiva para uma organização, contudo pelo fato do ambiente atualmente ser mais dinâmico, as organizações que tiverem a capacidade de perceber as ameaças e oportunidades de seus mercados, e as que melhor adequarem seus recursos conseguem um desempenho superior aos seus concorrentes. Como uma firma pode aplicar seus conhecimentos acumulados e suas habilidades desenvolvidas objetivando tornar-se inovadora, potencializando o seu desempenho?

$\mathrm{Na}$ área de pesquisa sobre estratégia organizacional, os estudos de capacidades dinâmicas, em especial as capacidades inovativas, pode-se considerar um campo ainda pouco pesquisado e em busca de sua consolidação, não existindo um modelo mais utilizado e que pode se generalizar a diversos cenários, o que vem ao encontro de Boava et al. (2012).

Ao estudarem uma escala de diferenciação de serviços na hotelaria executiva, sugerem os autores Castro Junior, Gonçalo e Rossetto (2014) que se adapte esta escala objetivando sua utilização em futuras pesquisas, independente do setor que a se estudar. Como resultante desta pesquisa, esta escala mostra três dimensões: Pessoas, Processo e Ambiente físico. Poderia a diferenciação de serviços mediar a relação de outra capacidade dinâmica com desempenho?

Poderia a capacidade inovativa possuir relação positiva e significativa, com a diferenciação de serviços? Existe predição entre as relações das capacidades inovativas, diferenciação de serviços e o desempenho no setor supermercadista em Santa Catarina?

Considerando essas questões, fica assim definida a questão problema que norteará este ensaio teórico: Qual a relação entre as capacidades inovativas, diferenciação de serviços e o desempenho nos supermercados de Santa Catarina?

\section{FUNDAMENTAÇÃO TEÓRICA}

Tometich, Fracasso e Zen (2014) afirmam que há existência de uma gama de abordagens no que tange as capacidades dinâmicas, relacionando-as com o processo de gestão da inovação. Desta maneira, podese encontrar, na literatura, 'capacidade' como sendo uma habilidade humana (Penrose, 1959; Becker, 1962; Barney, 1991); um conjunto de habilidades, experiências e conhecimentos encontrado em uma organização (Richardson, 1972). Estas perspectivas convergem em um sentido ao referir-se sobre a possibilidade das organizações desenvolverem suas operações de maneira competitiva, empregando conhecimento, experiência e habilidades para oferecer soluções a partir das oportunidades percebidas no mercado, gerando valor para atender a anseios dos consumidores. Desta forma, as capacidades permitem que as organizações se desenvolvam em um cenário dinâmico no mercado, dentre as abordagens teóricas relevantes, destaca-se as capacidades dinâmicas.

\subsection{Ambiente dinâmico}

Picoli e Takahashi (2016) explicam que as organizações estão inseridas em um ambiente dinâmico, onde as firmas agem em permanente estado de necessidade em moldar, e fortalecer seus processos, concedendo-lhes a sobrevivência e a respectiva evolução. Contudo, é necessário estar monitorando e identificando as oportunidades neste cenário que se está inserida, agindo na forma de fornecedor de inteligência ao disponibilizar alternativas para adaptar e aperfeiçoar suas atividades desenvolvidas no ambiente empresarial (Liao, Welsch \& Stoica, 2003; Jansen, Van Den Bosch \& Volberda, 2005; Lichtenthaler, 2009).

A partir desta premissa, Barney e Hesterly (2007), ambiente dinâmico, e mutável, explica que se pode visualizar a gestão estratégica como uma gama de opções, desenvolvidas de maneira sequencial objetivando o aumento das opções em gerar a vantagem competitiva. Conceituam que a administração estratégica começa na sequência da missão empresarial e perpassa ao longo da vida organizacional, que se encontra em eterna e as devidas adaptações ao mercado. Afirmam ainda, que a maior parte das firmas busca rever constantemente seus objetivos, adequando-os a novas configurações de mercado, para interagir da melhor forma com o seu ambiente.

Para Dalfovo, Machado, Wruck e Silva (2015) a firma deve optar pela estratégia que melhor a atenderá em um ambiente dinâmico, dentro do seu contexto, refletindo no ambiente interno, ou seja, o ambiente controlável.

De acordo com Silveira-Martins e Tavares (2014), pode-se afirmar que o ambiente no qual a firma está implantada é fonte ininterrupta de incertezas, podendo influenciar o desempenho da organização, 
evidenciando desta forma constante necessidade que o tomador de decisões, tem de desenvolver sua percepção e interpretação do ambiente com a maior eficácia e efetividade.

Pavão (2012) enfatiza que na literatura científica discute-se sobre o contexto da organização e o ambiente com uma variedade de relações. Em ambientes diferentes, há informações que podem interessar não apenas às empresas, assim como aos pesquisadores (Child, 1972; Mintzberg, 1979; Dess \& Beard, 1984) na busca de analisar, interpretar e compreender a relação que há entre o ambiente, as organizações e as categorias das empresas que as formam. Dentre os links efetivos entre as firmas e o seu ambiente, encontram-se as incertezas, podendo ser associadas a uma variedade gama de fatores.

Podem-se destacar as três dimensões de um ambiente: i) dinâmica, ii) complexa e iii) munificente, cada dimensão sendo possuidora de concepções próprias, seus fatores individuais e seus atributos. Destacando a historicidade disponível na literatura sobre firmas e seu ambiente (Dill, 1958; Burns \& Stalker, 1961; Duncan, 1972; Silveira-Martins \& Tavares, 2014). No Quadro 1, pode-se verificar a contribuição dos pesquisadores sobre as dimensões do ambiente, os temas abordados e as dimensões encontradas.

\subsection{Capacidades Dinâmicas}

Os autores Escobar (2012), Meirelles e Camargo (2014), Cabral, Coelho, Coelho e Costa (2015) e Vicente, Abrantes e Teixeira (2015), verificam que a evolução das Capacidades Dinâmicas percorre pelas contribuições de uma gama de teorias. Podendo-se destacar: i) teoria evolucionária (Schumpeter, 1934; Nelson \& Winter, 1982); ii) teoria da aprendizagem e de competências organizacionais (Prahalad \& Hamel, 1990), iii) custos de transação (Coase, 1937), iv) teoria da agência (Jensen \& Meckling, 1976), v) Teoria contingencial (Boyd, 1995), vi) teoria institucional (Zucker, 1987), e como destaca por diversos autores desta evolução, viii) da visão baseada em recursos - RBV (Barney, 1991). Na Figura 1, pode-se verificara um quadro resumo da evolução da teoria até chegar na teoria da capacidade dinâmica.

Figura 1 - Evolução da abordagem das Capacidades Dinâmicas

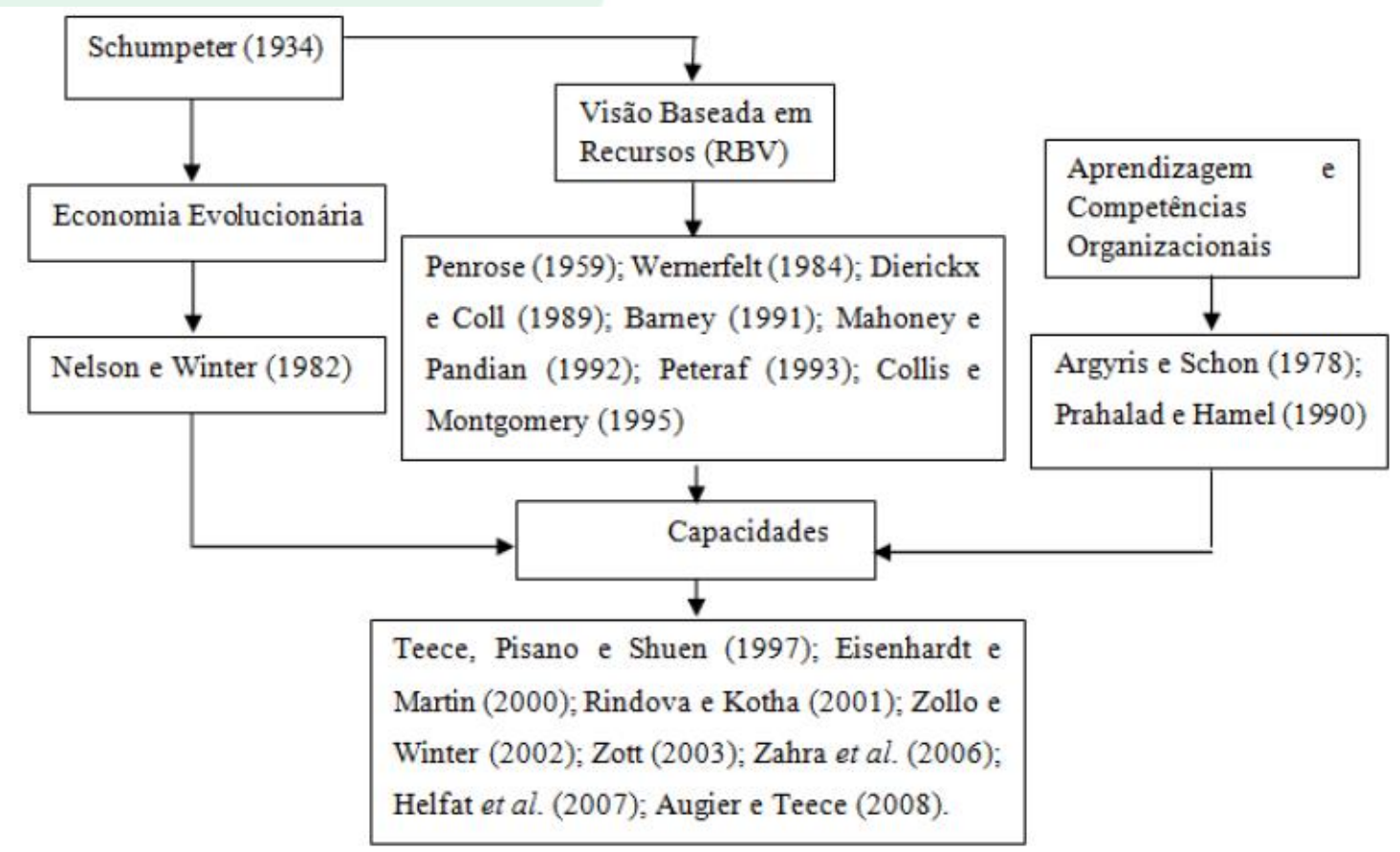

Fonte: Escobar (2012)

Ao pesquisar as capacidades dinâmicas, os autores Peteraf, Di Stefano e Verona (2013), realizaram uma pesquisa bibliométrica sobre o tema e destacam os autores mais citados.

Para Costa e Porto (2015) conviver e explorar as mudanças é um esforço inerente à atividade empresarial, no entanto, para sobreviver e prosperar sob condições de mudança, as empresas devem desenvolver 'capacidades dinâmicas' para criar, ampliar e modificar as formas pelas quais sobrevivem (Teece, 2007; Helfat, Finkelstein e Mitchell, 2007).

Tometich, Fracasso e Zen (2014) informam que as capacidades dinâmicas dizem respeito a uma abordagem que assume o cenário em que ocorrerão 
mudanças contínuas, onde as organizações devem permanentemente se ajustar, objetivando conseguir a manutenção da sua vantagem competitiva (Teece, Pisano \& Shuen, 1997; Eisenhardt \& Martin, 2000; Winter, 2003; Teece, 2007).

Silveira-Martins e Tavares (2014) afirmam que as competências dos gestores têm sido pesquisadas e caracterizadas pela literatura científica em estratégia, as capacidades dinâmicas (Teece \& Pisano, 1994; Zollo \& Winter, 2002; Andreeva \& Chaika, 2006). Tais competências diferenciam-se como sendo: gerencial, mercadológica e tecnológica.

Meirelles e Camargo (2014) apresentam as capacidades dinâmicas como sendo um ramo de pesquisa em administração. A partir das pesquisas bibliométricas realizadas por Barreto (2010), Stefano, Peteraf e Verona (2010) e Vogel e Gutel (2013), contendo os temas pesquisados, destacando: i) gerenciamento estratégico; ii) empreendedorismo, iii) marketing, iv) recursos humanos, v) operações, vi) sistema de informação. (Nelson, 1991; Teece \& Pisano, 1994; Teece et al. 1997; Dosi, Nelson \& Winter, 2000).

Ainda de acordo com Meireles e Camargo (2014), de maneira mais pragmática, a capacidade dinâmica é relevante principalmente nos cenários de uma economia globalizada e dinâmica, onde as alterações tecnológicas são instantâneas e sistêmicas (Teece, 2007, 2009).

\subsection{Capacidade Inovativa}

Cabral et al. (2015), a partir de Verhees e Meulenberg (2004), afirmam a existência de uma gama de interpretações para o termo 'inovação'. Ao passar do tempo, a capacidade inovativa pode ser conceituado como sendo entendido o processo do desenvolvimento de novos itens, assim como de processos. A capacidade inovativa tem sido abordada a níveis setoriais, por região geográfica, tamanhos distintos das firmas, e em seus níveis de projetos.

Vicente et al. (2015) confirmaram o que havia sido informado por Teece (2007), que as empresas enfrentam forte concorrência e rápidas mudanças tecnológicas, uma concorrência global, onde os ciclos de vida dos produtos estão cada vez mais curtos, valorizando a pesquisa e desenvolvimento de produtos, e da facilidade de imitar as organizações inovadoras, como forma de garantir a sua competitividade e a sua sobrevivencia a médio e longo prazo (Yam et. al., 2004; Chadha, 2009).

Shan e Zhang (2009) Yam et al., (2010) Wallin et al., (2011) e Golovko e Valentini (2011), comentam que uma vez que as empresas com capacidades inovativas podem obter acesso ao conhecimento ainda não disponível em seu ambiente interno, eles podem melhorar o processo de aprendizagem interno, que é a base da inovação que culmina com o melhor desempenho global.

Os autores Yalcinkaya et. al. (2007) afirmam que as organizações que continuadamente inserem novos produtos, assim como novos serviços, objetivando atender às necessidades e desejos de seus clientes, resultam como principal indicador a consecução de um desempenho superior. Esta afirmação corrobora com o que já afirmavam Sandvik e Sandvik (2003), que com a capacidade inovativa afeta três elementos de mercado: i) disponibilidade de um preço premium, o crescimento das vendas e a capacidade de utilização. Portanto, a inovação pode ser esperado para levar a um desempenho superior.

Lawson e Samson (2001), Calantone et. al., (2002) e Guan e Ma, (2003) como resultado de suas pesquisas, encontraram que com o desenvolvimento da capacidade inovativa, aumenta a probabilidade da organização integrar as suas capacidades essenciais para efetivamente estimular o desenvolvimento de produtos inovadores e o aumento de seu desempenho organizacional.

Escobar (2012) ao abordar a capacidade inovativa apresenta o resultado da pesquisa de Jambulingam et. al. (2005) que procurou operacionalizar a capacidade inovativa da firma utilizando-se das medidas atreladas à cultura. Sendo as dimensões propostas pelos pesquisadores: i) estímulo/recompensa para inovar, ii) geração, iii) seleção de ideias, e aproveitamento/tratamento/implementação de ideias. Com outra abordagem Liao, Fei e Chen (2007) pesquisaram qual a relação: i) aquisição de conhecimento, ii) capacidade de absorção, iii) capacidade de inovação em indústrias de Taiwan. No que tange a capacidade inovativa, a hipótese testada não se foca somente ao produto e processo, mas aborda à tecnologia e gestão.

Yesil, Koska e Büyükbese (2013) investigaram a relação entre a capacidade de inovação e o desempenho inovador das empresas. A partir de Liao et. al., (2007) a pesquisa abordou à capacidade inovativa alcançar uma melhoria global de seu desempenho. Lawson e Samson (2001) argumentou que as empresas excelentes que investirem e estimularem as capacidades inovativas, executam processos de inovação eficazes, levando a inovações em novos produtos, serviços e processos, e resultados de desempenho de negócios superiores.

\subsection{Diferenciação de Serviços}

Castro Junior et al. (2014) afirmam que existe abundante literatura científica que destacam a importância da diferenciação de serviços para a estratégia das organizações, emergindo a discussão sobre como executar a mensuração da diferenciação em serviços.

De acordo com Levitt (1980) uma firma ao objetivar uma posição vantajosa na competição no mercado, deve conseguir buscar a diferenciação seja em produtos ou serviços. Ressalta-se que os serviços devem ser diferentes para quem compara, não somente em ofertas, assim como nos produtos. 
A diferenciação, deve se dar por meio da qualidade do atendimento. Em decorrência deste entendimento, o setor de serviços deve focar seus esforços em compreender as exigências por parte dos consumidores; as configurações dos serviços que vão ao encontro dos desejos e das expectativas dos compradores / usuários de serviços (Lovelock, 1983; Grönroos, 1998, 2003; Parasuraman, Zeithaml \& Berry, 1985, 1988, 1991; Bitner, Booms \& Tetreault, 1990; Cronin \& Taylor, 1992).
Booms e Bitner (1981) indicam que o principal desafio aos gestores no que tange ao marketing de serviços é pensar criativamente sobre a estratégia do seu Marketing Mix. Expandido o composto para serviços, que são normalmente produzidos e consumidos simultaneamente na unidade produtiva na empresa, interagindo diretamente com o pessoal da empresa e nos processos de produção. O Quadro 1 apresenta a compreensão de cada uma das dimensões da diferenciação de serviços.

\begin{tabular}{||l|l||}
\hline Dimensão & \multicolumn{1}{c|}{ Compreensão da dimensão } \\
\hline Pessoas & $\begin{array}{l}\text { São considerados todos os agentes que desempenham um papel no } \\
\text { processo de execução de um serviço e, neste sentido, influenciam as } \\
\text { percepçóes do comprador, nominalmente, os funcionários da empresa, o } \\
\text { cliente e outros clientes no ambiente de serviços. }\end{array}$ \\
\hline Processos & $\begin{array}{l}\text { São os procedimentos, mecanismos e os roteiros efetivos de atividades } \\
\text { através dos quais o serviço é executado - os sistemas de execução e de } \\
\text { operação dos serviços. }\end{array}$ \\
\hline Ambiente & $\begin{array}{l}\text { São as evidências fisicas no quais os serviços são executados e onde a } \\
\text { empresa interage com o cliente, assim como qualquer componente } \\
\text { tangível que facilite o desempenho ou a comunicação dos serviços. }\end{array}$ \\
\hline
\end{tabular}

Quadro 1 - As Dimensões da diferenciação (serviços). Fonte: adaptado de Booms e Bitner (1981)

Corroborando com a teoria de Booms e Bitner (1981), Zeithaml e Bitner (2003) afirmam que os 4Ps tradicionais do composto de marketing ou marketing mix, Produto; Preço; Praça (ponto de distribuição) e Promoção (composto de comunicação), não são suficientes para o setor de serviços. Para se possibilitar a diferenciação dos serviços.

Apoiado com o autor anterior, este quadro demonstra como a equipe de colaboradores e seus programas de desenvolvimento e treinamento, associados a um processo eficaz e com boa atmosfera de loja, possibilitam uma melhor percepção de uma diferenciação de serviços.

Da mesma forma que o composto mercadológico tradicional com os seus 4Ps, o composto de serviço são combinados e serão abordados com mais profundidade nos próximos tópicos. Pessoas, Processo e Ambiente, também denominado como evidências físicas, elementos tangíveis, aqui sempre tratados como Ambiente físico ou atmosfera de loja.

\subsubsection{As dimensões da diferenciação de serviços}

Diversos autores, como Lehtinen e Lehtinen (1991), Watson, Pitt e Kavan (1998), Nielsen e Host (2000), Sheppard (2003), Lee (2005), Ibáñez, Hartmann e Calvo (2006), Castro Júnior et al. (2014) relacionaram as três dimensões da diferenciação de serviços são: i) qualidade física (ambiente de loja); ii) qualidade interativa e comunicação (pessoas); e iii) qualidade dos processos estão relacionados com o desempenho superior da firma. Neste presente projeto de tese serão expostas na sequência, as dimensões de diferenciação de serviços: Pessoas, Processos, Ambiente Físico.

\subsubsection{Dimensão - Pessoas}

Willborn (1986), Shrednick, Shutt e Weiss (1992), Ross (1995) e Dickens (1996), Riesenberger (1998), Haring e Mattsson (1999) ao estudarem empresas de serviços, afirmam que a diferenciação de prestadores de serviços está no treinamento/capacitação das pessoas, como forma de entregar a qualidade desejada e a garantia de um padrão organizacional, da administração dos seus serviços, e da imagem da firma, diferenciando-se de seus concorrentes.

No varejo esta imagem é percebida pelo posicionamento de mercado. De acordo com os pesquisadores Berry (1982) e Mason, Mayer e Wilkimson (1993) para decidir qual posicionamento, deve-se definir: i) Quais segmentos existentes estão a disposição?; ii) Qual segmento existe espaço?; iii) Para se ocupar o espaço, não se vende um produto, vende-se a empresa; iv) a loja passa a ser a marca. Desta forma a imagem da empresa varejista, busca um posicionamento claro, como forma de contribuir 'fortemente' para o sucesso da empresa varejista.

No setor de varejo, uma das formas de se diferenciar é por meio do atendimento e serviço;s ao 
consumidor. Autores como Zeithaml, Parasuraman e Berry (1990), Brown e Lam (2008), Dunne, Lusch e Carver (2011) que além de desenvolver um bom relacionamento pessoal entre o varejista e seu consumidor, este fator fundamental de se diferenciar de seus concorrentes, apresentam uma importante vantagem competitiva.

Dentre os diferentes níveis de serviços, Kunz (1998) apresenta alguns serviços para o autosserviço, como é o caso de supermercados, como horário de funcionamento, caixas, embalagens, entregas em domicílio, papel do colaborador, processo de compras na loja, trocas e devoluções, treinamento do colaborador e serviços especiais.

Segundo Parente e Barki (2014), os varejistas iniciam o processo de reconhecimento que o capital humano e seu profissionalismo possuem relação com o desempenho superior. Investimentos em programas de treinamento e capacitação profissional é a forma para se diferenciar de seus concorrentes. Pessoas mais qualificadas, com formação acadêmica para funções de compradores e gerentes de loja ou departamentos passam a ter maior relevância no setor de varejo, em especial nos supermercados.

Sharma e Levy (1995), Kunz (1998) explicam quais habilidades devem ser desenvolvidas para se apresentar a diferenciação de serviços no varejo. Os atributos e habilidades a serem desenvolvidas: i) Comunicabilidade; ii) Aparência pessoal; iii) Personalidade, iv) Conhecimento; v) Atitude; vi) Saber ouvir; vii) Similaridade e viii) Adaptabilidade.

\subsubsection{Diferenciação de Serviços - Processos}

Em estudos sobre a diferenciação e a qualidade de serviços , os pesquisadores como Oakland (1986), Edvardsson (1988), Edvardsson e Olsson (1996), Karatepe, Avci e Arasli (2004), Chang (2008) e Barrutia, Charterina e Gilsanz (2009), ultimaram ao afirmarem que durante o processo a atenção deve ser máxima, por se considerarem fundamental na diferenciação dos serviços, e conseguirem vantagens sobre os seus concorrentes. Uma vez que, é durante os processos que o consumidor percebe se a sua expectativa do serviço, ao serviço almejado, compararam com os serviços da concorrência. Normalização e padronização nos serviços, padrão de execução, tempo de processo, eficácia dos processos, planejamento do design de serviço, são considerados elementos fundamentais para a diferenciação de serviços,desta forma entende-se o processo como diferencial na prestação de serviços.

Kunz (1998) estudou os diferentes níveis de serviços, concluindo que representam uma vantagem competitiva, sendo uma forma eficaz de se diferenciar os serviços de seus concorrentes. Dunne et al. (2011) verificaram que além dos níveis de serviços, os tipos de serviços são importantes para a diferenciação de serviços.
Mason et al. (1993), Dunne et al. (2011), Parente e Barki (2014), destacam o processo de sistema de informação de marketing, para melhor gestão dos indicadores de desempenho das empresas varejista, como um dos importantes fatores de diferenciação e sucesso varejista. Destacam a automação nos sistemas de informação no varejo, tecnologia eletrônica do código de barra, automação comercial e a tecnologia eletrônica de rádio frequência para exemplificar fatores de diferenciação da oferta varejista em seus processos.

Para Parente e Barki (2014), dentre os processos para o sucesso dos varejistas, o modelo dos sete estágios do processo de compra de Blackwell, Miniard e Engel (2005), uma vez que o autosserviço não existe o contato com a equipe de venda.

Castro Junior et al. (2014) afirmam que ao executar corretamente o planejamento dos processos internos, e a excelência em sua execução, os processos desempenham papel fundamenta para a diferenciação dos serviços.

\subsubsection{Diferenciação de Serviços - Ambiente}

Para Watson, Pitt e Kavan (1998), o ambiente além de propiciar a execução dos serviços, tangibiliza o serviço aos olhos do consumidor, que poderá perceber a diferenciação entre os seus competidores. Llosa, Chandon e Orsingher (1998) destacam que a dimensão tangibilidade (evidências físicas) é claramente percebida pelos clientes, e é seguida pela dimensão da empatia. Nas conclusões dos autores, o ambiente é a principal dimensão utilizada pelos consumidores para diferir os seus fornecedores de serviços.

Kunz (1998) e Dunne et al. (2011) pesquisaram os níveis de serviços e tipos de serviços, afirmando que alguns elementos do ambiente físico entregam uma vantagem competitiva ao varejista, ao apresentar elementos diferenciais como, estacionamento, merchandising (no ponto de venda), transporte gratuito, serviços de transação, experiência com o produto (degustações e demonstrações), espaço de embalagens e empacotamentos e presentes, disponibilidade de mercadorias, serviços de entregas, montagens e instalações, espaço para trocas e devoluções.

Sá e Marcondes (2010) estudaram o varejo de luxo feminino, ao abordarem aspectos da apresentação externa das lojas e ambiente interno, basearam a pesquisa nas afirmações de Levy e Weitz (2000), Schmid (2003) e Pianaro e Marcondes (2008), na qual afiançavam que uma empresa que comercializa artigos de alto padrão (luxo) o ambiente é fundamental para tangibilizar ao cliente a imagem desenvolvida pela marca, corroborando a afirmação de nas estratégias de marketing. Portanto o ambiente da loja é desenvolvido para entregar a diferenciação de serviços, com adequação dos elementos de apresentação, layout e área de exposição.

Parente e Barki (2014) defendem a ideia que diversos elementos influenciam a apresentação interna 
da loja, criando um melhor ambiente de loja. A atmosfera da loja auxilia a estimular os consumidores no processo de compra. Elementos como cores ou sonorização provocam os sentidos. Sinalizações e equipamentos auxiliam a comunicação, largura dos corredores e climatização entregam conforto aos compradores. No Quadro 4 apresentam-se elementos internos e as funções na atmosfera de loja.

\subsection{Relação entre construtos e Proposições}

Lee e Kelley (2008) informam que na literatura sobre as capacidades dinâmicas de inovação, encontram-se domínios elevados de incerteza, o que produz pouca tranqüilidade para as organizações, que buscam melhorar a sua capacidade inovativa. Afirmam os autores que as práticas dos gestores de projeto de inovação são um componente chave das capacidades dinâmicas de uma organização orientada para a inovação. Afirmam que as práticas de gestão incluem a implantação de recursos empreendedoras, desenvolvimento de habilidades e motivação.

Ainda de acordo com Lee e Kelley (2008), sugerem os autores que as capacidades dinâmicas são úteis para o contexto de inovação. No entanto, não está claro o que compõe capacidades dinâmicas. Há um consenso de que as capacidades dinâmicas não são processos de rotina. Dada a necessidade de acesso ao conhecimento específico da situação, e adaptar-se a resultados imprevisíveis, simplesmente capacitar líderes de projeto e proporcionando um ambiente ideal não será suficiente. Propõe que as capacidades dinâmicas não são rotinas, mas compreendem que envolve primeiramente práticas de gestão, logo, a seleção de gestores que assumem a tarefa principal de montagem e integração dos recursos necessários para criar inovações.

$\mathrm{Fu}$ (2008) encotrou em sua pesquisa que a capacidade de inovação sofre efeitos da concorrência. A concorrência de mercado pode também ser vista como uma espada de dois gumes, em termos do seu efeito sobre a inovação. Argumenta-se que a falta de concorrência no mercado vai dar origem à ineficiência e resultar em atividade inovadora lenta. Por outro lado, o poder de reivindicação monopólio schumpeteriana tradicional torna mais fácil para as empresas adequadas para os retornos de inovação e, desta forma, oferece o incentivo para investir em inovação e novos serviços. Cabendo aos gestores desenvolver estratégia para a vantagem competitiva sobre os concorrentes.

Lages, Silva e Styles (2009) pelos resultados de sua pesquisa, indicam que os gerentes devem investir em capacidades de gestão de relacionamento para melhorar a inovação de produto e qualidade do produto, que por sua vez leva a melhoria de desempenho.

Çakar e Ertürk (2010) afirmam que a aversão à incerteza está relacionada com a capacidade de inovação. Os resultados também revelam que os gerentes devem se concentrar em práticas de gestão participativa para promover a capacidade de inovação.

O’Connor (2008) comenta que a capacidade de inovação propõe-se requer a interação de múltiplos elementos de um sistema de gestão que excedem em muito a complexidade de rotinas simplesmente operacional, que formam a base da teoria da capacidade dinâmica. Sete elementos específicos de um sistema de gestão são propostos que poderiam incluir uma capacidade dinâmica, se visto como um sistema integrado: i) uma estrutura organizacional identificável; ii) Interface de mecanismos; iii) Processos exploratórios; iv) competências necessárias; v) governança e mecanismos de tomada de decisão; vi) métricas de desempenho adequados; e vii) uma cultura apropriada e contexto de liderança. Cada um destes é elaborado com base no que sugerir tanto teoria e observação. Seus relacionamentos são discutidos para identificar esses elementos como necessária e suficiente para a construção de uma capacidade de inovação sustentável

De encontro com Gurkov (2006), a pesquisa de Akman (2008), afirma que a inovação é fator chave de sucesso competitivo. As relações entre orientação para o mercado, estratégia de inovação, capacidade de inovação estão relacionadas positivamente com o desempenho e o sucesso da inovação em países em desenvolvimento.

Siqueira e Cosh (2008) vão além ao concluírem que quanto maior a capacidade de inovação, maior a propensão ao desempenho superior.

Os autores Aulawi, Sudirman, Suryadi e Govindaraju (2009) afirmam que a capacidade dos empregados para inovar é um fator significativo para uma empresa para sobreviver na competição.

Além disso, Lages, Silva e Styles (2009) revelam que seus resultados, embora a qualidade do produto é um aspecto crítico nos mercados internacionais, tanto a inovação do produto e desempenho possuem um papel maior na melhoria do desempenho econômico.

De acordo com Çakar e Ertürk (2010), reconhece-se assim que a vantagem competitiva pode ser obtida com uma força de trabalho de alta qualidade que permite que as organizações para competir com base na qualidade e inovação, melhorando o desempenho organizacional.

Proposição 1: Capacidades inovativas têm relação positiva com desempenho.

Tsai (2006) o ambiente físco (estrutura) e capacidade inovativa possui uma relação positiva e significativa.

Afirmam Jorgensen e Kofoed (2007) que o aprendizado das pessoas possui relação positiva com a capacidade inovativa.

Os pesquisadores Aulawi, Sudirman, Suryadi e Govindaraju (2009) afirmam que os esforços eficazes para desenvolver a capacidade dos empregados para inovar em uma empresa é através do desenvolvimento de partilha de conhecimento, uma vez que através 
desse conhecimento atividade pode ser disseminada, implementada e desenvolvida. Ao estimular as pessoas a pensar mais criticamente e de forma mais criativa, de modo que, finalmente, pode produzir conhecimento novo que é benéfico para a empresa. Impacto positivo na capacidade de inovação.

Para os autores Lages, Silva e Styles (2009) para entender como um conjunto de recursos (Pessoas, Processos e Evidências físicas), relacionam-se com o desempenho.

A capacidade inovativa para Dervitsiotis (2010) perpassa pelos processos organizacionais, para gerar diferenciação de serviços e melhor desempenho.

A inovação tem sido definido, de acordo com Çakar e Ertürk (2010) de várias maneiras. No entanto, é geralmente definida como não só a concepção de um novo produto ou serviço (ou um produto ou serviço muito melhor), mas também como a interposição de sucesso do novo produto ou serviço para o mercado. Assim, a capacidade de inovação da empresa é a sua capacidade de mobilizar o conhecimento, possuído por seus empregados (Kogut \& Zander 1992), e combinálo para criar novos conhecimentos, resultando em produto e/ou inovação de processo.

Afirma Gurkov (2011) que novos produtos são geralmente a parte mais visível dos processos de inovação; na maioria dos casos a necessidade de desenvolver e lançar novos produtos serve como o principal impulso para inovações em processos e sistemas.

Cabral et. al. (2015), a partir de Verhees e Meulenberg (2004), afirmam a existência de uma gama de interpretações para o vocábulo 'inovação'. Ao passar do tempo, a capacidade inovativa pode ser conceituado como sendo entendido o processo do desenvolvimento de novos itens, assim como de processos

Proposição 2: Capacidades inovativas têm relação positiva com Diferenciação de Serviços na dimensão Processos.

Proposição 3: Capacidades inovativa têm relação positiva com Diferenciação de Serviços na dimensão Pessoas.

Proposição 4: Capacidades inovativa têm relação positiva com Diferenciação de Serviços na dimensão Ambientes.

Martins (2015) destaca que os diferenciadores representam outra dimensão muito importante da estratégia (Hambrick \& Fredickson, 2001). Encontra se associada à forma 'como' (Markides, 2001) a empresa competirá para conseguir bons resultados. Magretta (2012) esclarece que 'só competindo para ser única, para ser original, é que uma organização pode alcançar sustentadamente um desempenho superior'.

Booms e Bitner (1981) sugerem que as variáveis contraláveis tradicionais (Produto; Preço, Ponto de Distribuição e Promoção) não são o suficiente para entregar a diferenciação em serviços. Sendo responsável pela diferenciação de serviços as variáveis Pessoas, Processo e Evidências físicas.

Corroborando com a teoria de Booms e Bitner (1981), Zeithaml e Bitner (2003) afirmam que os 4Ps tradicionais do composto de marketing, Produto; Preço; Praça (ponto de distribuição) e Promoção (composto de comunicação), não são suficientes para o setor de serviço. Para se possibilitar a diferenciação dos serviços. Apresenta-se o composto de serviços expandido como sendo por Pessoas, Processo e Evidências físicas.

Diversos autores, como Lehtinen e Lehtinen (1991), Watson, Pitt e Kavan (1998), Nielsen e Host (2000), Sheppard (2003), Lee (2005), Ibáñez, Hartmann e Calvo (2006), Castro Júnior, Gonçalo e Rossetto (2014) relacionaram as três dimensões da diferenciação de serviços são: i) qualidade física (ambiente de loja); ii) qualidade interativa e comunicação (pessoas); e iii) qualidade dos processos estão relacionados com o desempenho superior da firma.

Willborn (1986), Shrednick, Shutt e Weiss (1992), Ross (1995) e Dickens (1996), Riesenberger (1998), Haring e Mattsson (1999), Castro Júnior (2013), Castro Junior et al. (2014) ao estudarem empresas de serviços, afirmam que a diferenciação de uma empresa de serviços está no treinamento/capacitação das pessoas, diferenciando-se de seus concorrentes.

Autores como Zeithaml, Parasuraman e Berry (1990), Brown e Lam (2008), Dunne et al. (2011) que além de desenvolver um bom relacionamento pessoal entre o varejista e seu consumidor, este é um fator fundamental de se diferenciar de seus concorrentes, consequentemente gerando uma importante vantagem competitiva.

Dentre os diferentes níveis de serviços, Kunz (1998) apresenta alguns serviços para o autosserviço, no caso de supermercados, o papel do funcionário, trocas e devoluções e treinamento do funcionário são destacados.

Dunne et al. (2011) verificaram que além dos níveis de serviços, os tipos de serviços são importantes para a diferenciação de serviços.

Em estudos sobre a diferenciação e qualidade de serviços pesquisadores como Oakland (1986), Edvardsson (1988), Edvardsson e Olsson (1996), Karatepe, Avci e Arasli (2004), Chang (2008) e Barrutia, Charterina e Gilsanz (2009), ultimaram ao afirmarem que durante o processo a atenção deve ser máxima, por se considerarem fundamental na diferenciação dos serviços, e conseguirem vantagens sobre os seus concorrentes.

Kunz (1998) estudou os diferentes níveis de serviços, concluindo que representam uma vantagem competitiva, sendo uma forma eficaz de se diferenciar os serviços de seus concorrentes.

Dunne et al. (2011) verificaram que além dos níveis de serviços, os tipos de serviços são importantes para a diferenciação de serviços. Definindo em 3 tipos. 
Mason et al. (1993), Dunne et al. (2011), Parente e Barki (2014), destacam diversos processos para a diferenciação de serviço. Sistema de informação de marketing, gestão dos indicadores de desempenho das empresas varejista, como um dos importantes fatores de diferenciação e sucesso varejista. Destacam a automação nos sistemas de informação no varejo, tecnologia eletrônica do código de barra, automação comercial e a tecnologia eletrônica de rádio frequência para exemplificar fatores de diferenciação da oferta varejista em seus processos.

Os processos para o sucesso dos varejistas, para Parenti e Barki (2014), o modelo dos sete estágios do processo de compra de Blackwell et al. (2005), uma vez que o autosserviço não existe o contato com a equipe de venda.

Para esclarecer o que é autosserviço, Ascar (2016) comenta que o autosserviço é uma técnica de venda surgida no começo do século 20 nos Estados Unidos, que revolucionou o varejo e permitiu o surgimento dos primeiros supermercados em 1930. Com ele desapareceram as mercearias com balcão separando o cliente da mercadoria. Assim os próprios consumidores selecionavam o que queriam e levavam para o caixa. Houve grande redução dos custos de pessoal e mais eficiência na operação. Foi uma revolução, o marco mais importante do varejo.

Complementando Sesso Filho (2003), conceitua autosserviço (self-service), sistema no qual o cliente escolhe os produtos sem a ajuda de funcionários, foi utilizada pela primeira vez em 1912 por comerciantes do Estado da Califórnia (EUA).

$\mathrm{O}$ primeiro supermercado norte-americano data de 1930. Chamava-se 'King Cullen Supermarket' e foi fundado por Michael Cullen. A adoção do autosserviço permitia a diminuição de custos com mão de obra, eliminava os pedidos por telefone e entregas em domicílio.

Para Watson, Pitt e Kavan (1998), o ambiente além de propiciar a execução dos serviços, tangibiliza o serviço aos olhos do consumidor, que poderá perceber a diferenciação entre os competidores.

Llosa, Chandon e Orsingher (1998) destacam que a dimensão tangibilidade (evidências físicas) é claramente percebida pelos clientes, e é seguida pela dimensão da empatia.

Kunz (1998) e Dunne et al. (2011) pesquisaram os níveis de serviços e tipos de serviços, afirmando que alguns elementos do ambiente físico entregam uma vantagem competitiva ao varejista. Diferenciais como, estacionamento, merchandising (ações no ponto de venda), transporte gratuito, experiência com o produto (degustações e demonstrações), espaço de embalagens e empacotamentos, disponibilidade de mercadorias, serviços de entregas, montagens e instalações, espaço para trocas e devoluções.

Sá e Marcondes (2010) ao abordarem aspectos da apresentação externa das lojas e seu ambiente interno, basearam a pesquisa nas afirmações de Levy e
Weitz (2000), Schmid (2003) e Pianaro (2008), na qual afiançava que o ambiente é fundamental para tangibilizar ao cliente a imagem desenvolvida pela marca. Portanto o ambiente da loja é desenvolvido para entregar a diferenciação de serviços.

Parente e Barki (2014) defendem a ideia que diversos elementos influenciam a apresentação interna da loja, criando um melhor ambiente de loja. Ao pesquisar a diferenciação no varejo, os autores afirmam que a apresentação da loja, tamanho, distribuição dos departamentos, padrão da apresentação interna, sua localização, ambientes dentro de shoppings centers são fundamentais para desenvolver a estratégia e tática da diferenciação.

Proposição 5: Diferenciação de Serviços na dimensão Processos tem relação positiva com o desempenho.

Proposição 6: Diferenciação de Serviços na dimensão Pessoas tem relação positiva com o desempenho.

Proposição 7: Diferenciação de Serviços na dimensão Ambientes tem relação positiva com o desempenho.

\subsection{MEDIAÇÃ̃O}

Tsai e Tsai (2010) examinam o impacto da capacidade inovativa no desempenho dos negócios. Por meio de um estudo empírico, revelou que a capacidade de inovação tem um efeito positivo significativo sobre o desempenho do negócio. O cluster de cadeia de valor (no tecido de clusters industriais) tem um efeito moderador sobre a capacidade e desempenho das empresas de inovação. Demonstrando que o efeito moderador em parques de ciência promoveu $o$ desempenho superior, quando modera a formação e construção de clusters industriais. Indicam que mais estudos devem ser realizados para verificar esta tendência para o futuro.

Withers, Drnevich e Marino (2011) apresentam um estudo que oferece insights para pesquisadores e gestores. Para os pesquisadores, ele fornece uma nova compreensão da moderação na idade das empresas para a alavancagem de recursos para inovação. Para os gestores, fornece um contexto que auxilia a identificação de táticas específicas ao melhor aproveitamento de duas grandes capacidades empresariais para o sucesso da inovação as capacidades de reconhecer oportunidades para a inovação e para combinar e organizar recursos complementares para explorar identificados oportunidades. Finalizam, apresentando a sugestão, e o desejo, que este estudo pode servir para motivar ainda mais a investigação e melhorar as práticas nesta área, com o auxília da moderação.

Gurkov (2011) Capacidade de inovação é amplamente definido como "a capacidade de transformar continuamente conhecimentos e ideias em novos produtos, processos e sistemas para o benefício da empresa e seus stakeholders". 
Castro Júnior (2013) e Castro Júnior et al. (2014) verificaram que amoderação da diferenciação de serviços possui relação positiva da capacidade mercadológica e o desempenho. Sugerem que novas relações devam ser testadas com a moderação da diferenciação de serviços.

Proposição 8: Capacidades inovativa têm relação positiva com o desempenho mediado pela diferenciação de serviços em sua dimensão Pessoas

Proposição 9: Capacidades inovativa têm relação positiva com o desempenho mediado pela diferenciação de serviços em sua dimensão Processos

Proposição 10: Capacidades inovativa têm relação positiva com o desempenho mediado pela diferenciação de serviços em sua dimensão Ambiente Após serem apresentadas as hipóteses a serem testadas, apresenta-se a metodologia proposta para o presente projeto de tese.

\section{CONCLUSÃO}

A discussão central deste ensaio teórico se suporta nas relações entre Capacidade inovativa, diferenciação de serviços como preditores do desempenho organizacional.

Neste cenário, da capacidade inovativa seria mediada pela diferenciação de serviços para se conseguir um desempenho superior. Cabendo ao gestor organizacional desenvolver as capacidades inovativas objetivando acesso ao conhecimento que ainda não está disponível em seu ambiente interno, melhorarndo desta forma o processo de aprendizagem organizacional, baseada na inovação que determinará em um desempenho superior.

Booms e Bitner (1981) afirmam que a diferenciação de serviços se adquirem por meio de 3 variáveis controláveis pela organização, Pessoas, Processos e Ambiente. Castro Junior et al. (2014) desenvolveram e validara uma escala de diferenciação de serviços na hotelaria executiva a partir das variáveis de Booms e Bitner (1981) e sugerem estes outras escalas sejam testadas e validadas em outros setores empresariais, o que pode contribuir para o refinamento das mesmas, tornando produtivo o seu uso em amplo número de estudos hipotéticos.

Neste ensaio teórico encontraram-se as relações entre a capacidade inovativa com Pessoas, Processos e Ambiente, elemento que formam a diferenciação de serviços, o que permite a proposição que a capacidade inovativa poderá ser mediada pela diferenciação de serviços, em estudo com o desempenho organizacional, possibilitando um desempenho superior.

Interpreta-se que a organização que consegue se diferenciar no mercado fazendo uso de suas habilidades internas e dos conhecimentos disponíveis junto aos seus stakeholders poderá conseguir direcionar-se ao mercado de forma que venha a obter melhores desempenhos e uma vantagem competitiva.
No que se refere a implicações gerenciais para o campo organizacional, sugere-se que a compreensão sobre práticas de gestão das relações com a capacidade inovativa e sua influência aos elementos da diferenciação de serviços, direcionando as estratégias das organizações proporcionando maior vantagem competitiva. Esta vantagem pode ser sustentada mediante a implementação de estratégias de criação de valor que são de grande dificuldade de serem implementadas por seus concorrentes.

\section{REFERÊNCIAS}

Akman, G. (2008). Innovative capability, innovation strategy and market orientation: an empirical analysis in turkish software industry. International Journal of Innovation Management, 12 (1): 69-111.

Andreeva, T. \& Chaika, V. (2006). Dynamic Capabilities: what they need to be dynamic? St. Petersburg State University. São Petersburgo: SPb. 2006.

Aulawi, H.; Sudirman, I.; Suryadi, K. \& Govindaraju, R. (2009) Knowledge Sharing Behavior, Antecedent and Their Impact on the Individual Innovation Capability. Journal of Applied Sciences Research, 5 (12): 2238-2246.

Barney, J. B. (1991). Firm resource and sustained competitive advantage. Journal of Management, 17 (1): 99-120.

Barney, J. B. \& Hesterly, W. S. (2007) Administração estratégica e vantagem competitiva. São Paulo: Pearson Prentice Hall.

Barrutia, J. M; Charterina, J. \& Gilsanz, A. (2009) Eservice quality: an internal, multichannel and pure service perspective. The Service Industries Journal. 29 (12): 1707-1721.

Becker. G. (1962). Investment in Human Capital: A Theoretical Analysis. Journal of Political Economy, 70 (5): 9-49.

Berman, B. \& Evans, J. R. (2009). Retail Management: A Strategic Approach, Pearson Education.

Berry, L. (1982). Retail Positioning Strategies for the 1980’s. Business Horizon, 25(6): 45-50.

Bitner, M. J; Booms, B. H. \& Tetreault, M. S. (1990) The Service Encounter: Diagnosing Favorable and Unfavorable Incidents. Journal of Marketing. 54 (1): 71-84. 
Blackwell, R. D.; Miniard, P. W. \& Engel, J. E. (2005) Comportamento do consumidor. 9. ed. São Paulo: Pioneira.

Boava, D. L. T.; Macedo, F. M. F. \& Sette, R. S. (2012) Contribuições do Ensaio Teórico para os Estudos Organizacionais. Anais do $7^{\circ}$ Encontro de Estudos Organizacionais. ANPAD. Curitiba, Brasil.

Booms, B. H. \& Bitner, M. J. (1981) Marketing strategies and organizational structures for service firms. In: J. Donnelly e W. R. (eds) Marketing of services, pp 47-51, Chicago: American Marketing Association.

Brown, S. \& Lam, S. K. A. A. (2008). Meta-Analysis of Relationships Linking Employee Satisfaction to Custumer Responses. Journal of Retailing, 84 (3): 243-255.

Burns, T. \& Stalker, G. M. (1961). The management of innovation. London: Tavistock Publications.

Cabral J. E. O.; Coelho, A. F. M.; Coelho, F. J. F. \& Costa, M. P. B. (2015). Capabilities, innovation, and overall performance in Brazilian export firms. RAM, Revista de Administração Mackenzie, 16 (3): 76-108.

Çakar, N. D. \& Ertürk, A. (2010). Comparing Innovation Capability of Small and Medium-Sized Enterprises: Examining the Effects of Organizational Culture and Empowerment. Journal of Small Business Management. 48 (3): 325-359.

Calantone, R. J.; Cavusgil, S. T. \& Zhao, Y. (2002) Learning Orientation, Firm Innovation Capability and Firm Performance. Industrial Marketing Management, 31(6): 515-524.

Castro Júnior, D. F. L. (2013) Estratégias para a hotelaria executiva catarinense: análise entre as capacidades mercadológicas, diferenciação de serviços e desempenho. Tese de Doutorado em Administração. Universidade do Vale do Itajaí, Biguaçu, Santa Catarina.

Castro Júnior, D. F. L.; Gonçalo, C. R. \& Rossetto, C. R. (2014). Diferenciação de serviços: construção e validação de escalas para mensuração. Revista de Administração FACES Journal, 13 (1): 46-64.

Chang, H. S. (2008) Increasing hotel customer value through service quality cues in Taiwan. The Service Industries Journal. 28 (1): 73-84.

Child, J. (1972). Organization Structure, Environmente, and Performance. Sociology. 6: 1227.
Costa, P. R. \& Porto, G. S. (2015). Capacidade dinâmica de cooperação nas multinacionais Brasileiras e seus fatores gerenciais determinantes. Revista Eletrônica de Negócios Internacionais ESPM, 10 (1): 57-69.

Cronin Jr, J. J. \& Taylor, A. S. (1992). Measuring Service Quality: a reexamination and a extension. Journal of Marketing, 56: 55-68.

Dalfovo, M. S; Machado, M. M; Wruck, J. \& Silva, G. V. (2015). Análise da disponibilidade dos recursos organizacionais no atendimento aos stakeholders no setor de serviços. Revista Interdisciplinar Científica Aplicada, 9 (1): 87-104.

Dervitsiotis, K. N. (2010) Developing full-spectrum innovation capability for survival and success in the global economy. Total Quality Management, 21 (2): 159-170.

Dess, G. G. \& Beard, D. W. (1984). Dimensions of organizational task environments. Administrative Science Quarterly. 29: 52 - 73.

Dickens, P. (1996). Human Service as service Industries. The Service Industries Journal. 16(1): 82-91.

Dill, W. R. (1958). Environment as an influence on managerial autonomy. Administrative Science Quarterly, 2(4): 409-443.

Dosi, G., Nelson, R. R. \& Winter, S. G. (2000) Introduction: the nature and dynamics of organizational capabilities. In G. Dosi, R. Nelson, \& S. G. Winter (Eds.). The nature and dynamics of organisational capabilities, pp 12-33. Oxford: Oxford Press.

Duncan, R. B. (1972). Characteristics of organizational environments and perceived environmental uncertainty. Administrative Science Quarterly, 17(3): 313-327.

Dunne, P.; Lusch, R. \& Carver, J. (2011). Retailing. 7. ed. Ohio: South Westrn Cengage Learning.

Edvardsson, B. (1988). Service Quality in Customer Relationships: A study of critical incidents in mechanical engineering companies. The Service Industries Journal. 8 (4): 427-445.

Edvardsson, B. \& Olsson, J. (1996). Key Concepts for New Service Development. The Service Industries Journal. 16 (2): 140-164.

Eisenhardt, K. \& Martin, J. (2000). Dynamic capabilities: What are they? Strategic Management Journal, 21 (10): 105-121. 
Escobar, M. A. R. (2012). Relação entre Capacidades dinâmicas, orientação empreendedora com o desempenho moderado pelo ambiente organizacional. Tese de Doutorado em Administração, Universidade do Vale do Itajaí, Biguaçu, Santa Catarina.

Fu, X. (2008). Foreign Direct Investment, Absorptive Capacity and Regional Innovation Capabilities: Evidence from China. Oxford Development Studies, 36 (1).

Golovko, E. \& Valentini, G. (2011). Exploring the complementarity between innovation and export for SMEs' growth. Journal of International Business Studies, 42: 362-380.

Grönroos, C. (1998). Marketing services: the case of a missing product. Journal of Business and Industrial Marketing. 13 (4/5): 322-338.

Grönroos, C. (2003). Marketing: gerenciamento e serviços. Rio de Janeiro: Elsevier.

Gurkov, I. (2006). Innovative Actions and Innovation (In)capabilities of Russian Industrial Companies An Extension of a Quasi-longitudinal Study. PostCommunist Economies, 23(4): 507-516 .

Hambrick, D. C. \& Fredrickson, J. W. (2001). Are you sure you have a strategy? Academy of Management Executive. 15 (4): 48-59.

Haring, M. D. \& Mattsson, J. (1999) A Linguistic Approach to Studying Quality of Face-to-Face Communication. The Service Industries Journal. 19 (2): 28-48.

Helfat, C. E.; Finkelstein, S. \& Mitchell, W. (2007). Dynamic Capabilities: Understanding Strategic Change. Oxford: Blackwell Publishing.

Ibáñez, V. A.; Hartmann, P. \& Calvo, P. Z. (2006). Antecedents of Customer Loyalty in Residential Energy Markets: Service Quality, Satisfaction, Trust and Switching Costs. The Service Industries Journal. 26 (6): 633-650.

Jambulingam, T.; Kathuria, R. \& Doucette, W. R. (2005). Entrepreneurial Orientation as a Basis for Classifi cátion within a Service Industry: The case of retail pharmacy industry. Journal of Operations Management, 23: 23-24.

Jansen, J. P.; Van Den Bosch, F. A. J. \& Volberda, H. W. (2005). Managing potencial and realized absorptive capacity: how do organizational antecedents matter. Academy of Management Journal, 48 (6): 999-1015.
Jorgensen, F. \& Kofoed, L. B. (2007). Integrating the development of continuous improvement and innovation capabilities into engineering education. European Journal of Engineering Education. 32 (2): 181-191.

Karatepe, O.M; Avci, T. \& Arasli, H. (2004). Effects of Job Standardization and Job Satisfaction on Service Quality: A Study of Frontline Employees in Northern Cyprus. Services Marketing Quarterly. 25 (3): 01-17.

Kunz, G. I. (1998). Merchandinsing: Theory Principles and Practice. New York: Fairchild Books.

Lages, L. F.; Silva, G. \& Styles, C. (2009) Relationship Capabilities, Quality, and Innovation as Determinants of Export Performance. Journal of International Marketing, 17 (4): 47-70.

Lawson, B. \& Samson, D. (2001). 'Developing Innovation Capability in Organizations: A Dynamic Capabilities Approach', International Journal of Innovation Management, 5 (3): 377-400.

Lee, H. \& Kelley, D. (2008). Building dynamic capabilities for innovation: an exploratory study of key management practices. $R \& D$ Management, 38 (2): 155-168.

Lee, J. (2005). Measuring Service Quality in a Medical Setting in a Developing Country: The Applicability of SERVQUAL. Service Marketing Quarterly. 27 (2): 1-14.

Lehtinen, U. \& Lehtinen, J .R. (1991). Two Approaches to Service Quality Dimensions. The Service Industries Journal. 11 (3): 287-303.

Levitt, T. (1980). Marketing Sucess Through Differentiation - of Anything. Harvard Business Review. 58 (1).

Levy, M. \& Weitz, B. (2000). Administração de varejo. São Paulo: Atlas.

Liao, J.; Welsch, H. P. \& Stoica, M. (2003). Organizational absorptive capacity and responsiveness: an empirical investigation of growth-oriented SMEs. Entrepreneurship Theory and Practice, 28 (1): 63-85.

Liao, S. H.; Fei, W. C. \& Chen, C. C. (2007). Knowledge sharing, absorptive capacity, and innovation capability: an empirical study of Taiwan's knowledge intensive industries. Journal of information science, 23: 340-359.

Lichtenthaler, U. (2009). Absorptive capacity, environmental turbulence, and the complementarity 
of organizational learning processes. Academy of Management Journal, 52 (4): 822-846.

Llosa, S; Chandon, J. L. \& Orsingher, C. (1998). An Empirical Study of SERVQUAL's Dimensionality. The Service Industries Journal. 18 (2): 16-44.

Lovelock, C. H. (1983). Classifying Services to Gain Strategic Marketing Insights. Journal of Marketing. 47: 9-20.

Lovelock, C. H. \& YIP, G. S. (1996). Developing Global Strategies for Service Businesses. California Management Review. 38 (2): 64-86.

Markides, C. (2001) Strategy as balance: from "EitherOr" to "And". Business Strategy Review. 12 (3): 110 .

Mason, J, B.; Mayer, M, L. \& Wilkinson, J. B. (1993). Modern Retailing: Teory and Practice. EUA: Irwin.

Meirelles, D. S. \& Camargo, Á. A. B. (2014). Capacidades Dinâmicas: O que são e como identificá-las? RAC - Revista de Administração Contemporânea, 18 (3): 41-64.

Meneghetti, F. K. (2011). O que é um Ensaio-Teórico? RAC - Revista de Administração Contemporânea, 15 (2): 320-332.

Mintzberg, H. (1979). An emerging strategy of "direct" research. Administrative Science Quartely, 24: 582589.

Nelson, R. R. (1991). Why do firms differ, and how does ite matter? Strategic Management Journal, 12 (2): 61-74.

Nielsen, J. F. \& Host, V. (2000). The Paht to service encounter Performance in public and private "bureaucracies". The Service Industries Journal. 20 (1): 40-60.

Oakland, J. S. (1986). Systematic Quality Management in Banking. The Service Industries Journal. 6 (2): 193-204.

O'Connor, G. C. (2008). Major Innovation as a Dynamic Capability: A Systems Approach. Journal of Product Innovation Management. 25 (4): 313330 .

Parasuraman, A.; Zeithaml V. A. \&Berry, L. L. (1991). Refinement and reassessment of the Servqual Scale, Journal of Retailing, 67 (4): 420-50.

(1985). A conceptual model of service quality and its implications for further research. Journal of Marketing, 49: 41-50.
(1988). Servqual: a multiple-item scale for measuring consumer perceptions of service quality. Journal of Retailing, 64 (1): 12-37.

Parente, J. \& Barki, E. (2014). Varejo no Brasil: Gestão e Estratégia. 2. ed. São Paulo: Atlas.

Pavão, Y. M. P. A. (2012). Capacidade de gestão dos stakeholders e o ambiente organizacional: relações e impactos no desempenho das cooperativas do Brasil. Tese de Doutorado em Administração. Universidade do Vale do Itajaí, Biguaçu, Santa Catarina.

Penrose, E. (1959). The Theory of the growth of the Firm. Oxford University Press.

Peteraf, M.; Di Stefano, G. \& Verona, G. (2013). The elephant in the room of dynamic capabilities: bringing two diverging conversations together. Strategic Management Journal, 34: 1389-1410.

Pianaro, L. \& Marcondes, R. C. (2008). Estratégias de Marketing no Mercado de Roupas e Acessórios de Luxo. Faces: Revista de Administração, 7 (1): 103122.

Picoli, F. R. \& Takahashi, A. (2016). Capacidade de Absorção, Aprendizagem Organizacional. RAC Revista de Administração Contemporânea, 20 (1): $1-20$.

Prahalad, C. K. \& Hamel, G. (1990) The core competence of the corporation. Harvard Business Review, 68 (3): 79-91.

Rebouças, G. M. (2008). O ensaio como reflexão metodológica para o campo jurídico. Anais do $13^{\circ}$ Encontro Nacional do CONPEDI - Salvador, pp 3196-3209, Florianópolis: Fundação Boiteux.

Richardson, G. B. (1972). The Organization of industry. Economic Journal. 82: 883-896.

Riesenberger, J. R. (1998). Executive Insights: Knowledge - The Source of Sustainable Competitive Advantage. Journal of International Marketing. 6 (3): 94-107.

Ross, G. F. (1995). Interpersonal Stress Reactions and Service Quality Responses among Hospitality Industry Employees. The Service Industries Journal. 15 (3): 314-331.

Sá, R. R. L. G. \& Marcondes, R. C. (2010). O ponto de vendas de produtos de luxo da moda de vestuário feminino no Brasil. Cadernos EBAPE, 8 (3): 531534. 
Sandvik, I. L. \& Sandvik, K. (2003). The impact of market orientation on product innovativeness and business performance. International Journal of Research in Marketing. 20 (4): 355-376.

Schmid, E. F. (2003). Varejo de moda - estratégias de marketing para diferenciação diante da concorrência. In: ANGELO, C. F. \& SILVEIRA J. A. G. Varejo competitivo, pp 107-133, São Paulo: Atlas.

Schumpeter, J. A. (1934). The Theory of Economic Development: An Inquiry into Profi ts, Capital, Credit, Interest, and the Business Cycle. Cambridge: Harvard University Pres.

Shan, W. \& Zhang, Q. (2009). Extension theory and its application in evaluation of independent innovation capability. Kybernetes. 38 (3): 457-467.

Sharma, A. \& Levy, M. (1995). Categorization of Costumers by Retail Salespeople. Journal of Retailing, 71(1): 71-81.

Sheppard, L. (2003). Network Development and application in Health Care: A Study of Service Quality. Services Marketing Quarterly. 24 (3): 4361.

Shrednick, H; Shutt, R. \& Weiss, M. (1992). Empowerment: Key to IS World-Class Quality. MIS Quarterly. 16 (4): 491-504.

Silveira-Martins, E. \& Tavares, P. M. (2014) Processo de Formulação de Estratégias: Capacidade Mercadológica, Incerteza Ambiental e Desempenho. Revista Organizações em Contexto (Online), 10: 297-322.

Siqueira, A. C. O. \& Cosh, A. (2008). Effects of product innovation and organisational capabilities on competitive advantage: evidence from uk small and medium manufacturing enterprises. International Journal of Innovation Management, 12 (2): 113-137.

Teece, D. J. (2007). Explicating dynamic capabilities: the nature and microfoundations of (sustainable) enterprise performance. Strategic Management Journal, 28 (13): 1319-50.

Teece, D. J. (2009). Dynamic capabilities \& strategic management. Oxford: Oxford University Press.

Teece, D. J.; Pisano, G. \& Shuen, A. (1997). Dynamic Capabilities and Strategic Management. Strategic Management Journal, 18: 509-533.
Teece, D. \& Pisano, G. (1994). The dynamic capabilities of firms: an introduction. Industrial and Corporate Change, 3 (3): 537-556.

Tometich, P; Fracasso, E. M. \& Zen, A. C. (2014). Reconfiguring the firm's capabilities for innovation. Proceedings of the 23th. International Management of Technology Anual Conference Science, Technology and Innovation in the age of Economic, Political and Security Challenges 2014. Miami, United States of America.

Tsai, M. T. \& Tsai, C. L. (2010). Innovation capability and performance in taiwanese science parks: exploring the moderating effects of industrial clusters fabric. The International Journal of Organizational Innovation, 2 (4): 80-103.

Tsai, Y. C. (2006). Effect of Social Capital and Absorptive Capability on Innovation in Internet Marketing. International Journal of Management, 23 (1).

Vale, G. M. V. \& Lopes, H. E. G. (2010). Cooperação e Alianças: Perspectivas Teóricas e suas Articulações no Contexto do Pensamento Estratégico. RAC - Revista de Administração Contemporânea, 14 (4): 722-737.

Verhees, F. J. H. M. \& Meulenberg, M. T. G. (2004). Market orientation, innovativeness, product innovation, and performance. Journal of Small Business Management, 42 (2): 134-154.

Vicente, M; Abrantes, J. L. \& Teixeira, M. S. (2015). Measuring innovation capability in exporting firms: the INNOVSCALE. International Marketing Review, 32 (1): 29-51.

Watson, R. T; Pitt, L. F. \& Kavan, C. B. (1998). Measuring Information Systems Service Quality: Lessons From Two Longitudinal Case Studies. MIS Quarterly. 22 (1): 61-79.

Willborn, W. (1986). Quality Assurance Audits and Hotel Management. The Service Industries Journal. 6 (3): 293-308.

Winter, S. (2003). Understanding dynamics capacidades. Strategic Management Journal, 24 (10): 991-995.

Withers, M. C;. Drnevich, P. L. \& Marino, L. (2011). Doing More with Less: The Disordinal Implications of Firm Age for Leveraging Capabilities for Innovation Activity. Journal of Small Business Management, 49 (4): 515-536. 
Yalcinkaya, G.,; Calantone, T. J. \& Griffith, D. A. (2007). An examination of exploration and exploitation capabilities: implications for product innovation and market performance. Journal of International Marketing, 15 (4): 63-93.

Yam, R. C. M.; Lo, W.; Tang, E. P. Y.; \& Lau, A. K. W. (2010). Technological Innovation Capabilities and Firm Performance, International Scholarly and Scientific Research \& Innovation, 4 (6): 1056-1064.

Yesil, S; Koska, A. \& Büyükbese, T. (2013). Knowledge Sharing Process, Innovation Capability and Innovation Performance: An Empirical Study. Procedia - Social and Behavioral Sciences, 75: 217-225.
Zeithaml, V. \& Bitner, M. J. (2003). Marketing de Serviços: a empresa com foco no cliente. 2. ed., Porto Alegre: Bookman.

Zeithaml; V; Parasuraman, A. \& Berry, L. L. (1990). Delivering Quality Service: Balancing Customer Perceptions and Expectations. New York: The Free Press.

Zollo, M. \& Winter, S. G. (2002). Deliberate Learning and the Evolution of Dynamic Capabilities. Organization Science, 13 (3): 339-351.

Zucker, L. G. (1987). Normal change or risk business: Institucional effects on the "hazard" of change in hospital organizations, 1959-79. Journal of Management Studies, 24 (6): 671-700. 